\title{
Fisioterapia dermatofuncional: radiofrequência e os efeitos no tratamento do rejuvenescimento da expressão da face
}

\author{
Dermatofunctional physiotherapy: radiofrequency and the effects on the rejuvenation of face expression treatment \\ Fisioterapia dermatofuncional: radiofrecuencia y efectos sobre el rejuvenecimiento del tratamiento de expresión facial
}

\begin{abstract}
RESUMO
Objetivo: Identificar, através de um levantamento bibliográfico, os efeitos da radiofrequência no rejuvenescimento facial. Métodos: Estudo de revisão integrativa, realizado no período de setembro a outubro de 2020. Pesquisados nos bancos de dados: LILACS, SciELO, Periódicos CAPES e PubMed. Os descritores utilizados e relacionados, com o operador booleno "and", em português e inglês foram: radiofrequência, expressão da face, pele Rejuvenescimento; Fisioterapia e Radio frequency; Face expression; Skin; Rejuvenation; Physiotherapy. Foram realizadas buscas publicações dos últimos 10 anos. Resultados: Após a leitura minuciosa dos artigos elegiveis, foram selecionados três artigos. Os autores relatam que os efeitos da radiofrequência são significativos na redução da extensão e profundidade das rugas e na melhora da aparência da flacidez pele, além de melhora perceptivel da expressão da face. Conclusões: Os efeitos da radiofrequência delinearam-se de maneira bastante evidente, principalmente no que diz respeito a melhora do tônus da pele e da diminuição das rugas.
\end{abstract}

DESCRITORES: : Radiofrequência; Expressão da face; Pele; Rejuvenescimento; Fisioterapia

\section{ABSTRACT}

Objective: To identify, through a literature review, the effects of radiofrequency on facial rejuvenation. Methods: Integrative review study, carried out from September to October 2020. Searched in the databases: LILACS, SciELO, CAPES Periodicals and PubMed. The descriptors used and related, with the Boolean operator "and", in Portuguese and English were: radiofrequency, facial expression, skin Rejuvenation; Physiotherapy and Radio frequency; Face expression; Skin; Rejuvenation; Physiotherapy. Searches for publications from the last 10 years were carried out. Results: After a thorough reading of the eligible articles, three articles were selected. The authors report that the effects of radiofrequency are significant in reducing the extension and depth of wrinkles and improving the appearance of sagging skin, in addition to a noticeable improvement in facial expression. Conclusions: The effects of radiofrequency were clearly outlined, especially with regard to improving skin tone and reducing wrinkles. DESCRIPTORS: Radio frequency; Face expression; Skin; Rejuvenation; Physiotherapy

\section{RESUMEN}

Objetivo: Identificar, a través de una revisión de la literatura, los efectos de la radiofrecuencia en el rejuvenecimiento facial. Métodos: Estudio de revisión integrativa, realizado de septiembre a octubre de 2020. Búsqueda en las bases de datos: LILACS, SciELO, CAPES Periodicals y PubMed. Los descriptores utilizados y relacionados, con el operador booleano "y", en portugués e inglés fueron: radiofrecuencia, expresión facial, rejuvenecimiento de la piel; Fisioterapia y Radiofrecuencia; Expresión facial; Piel; Rejuvenecimiento; Fisioterapia. Se realizaron búsquedas de publicaciones de los últimos 10 años. Resultados: Después de una lectura exhaustiva de los artículos elegibles, se seleccionaron tres artículos. Los autores informan que los efectos de la radiofrecuencia son importantes para reducir la extensión y profundidad de las arrugas y mejorar la apariencia de la piel flácida, además de una mejora notable en la expresión facial. Conclusiones: Los efectos de la radiofrecuencia fueron claramente delineados, especialmente en lo que respecta a mejorar el tono de la piel y reducir las arrugas.

DESCRIPTORES: Radiofrecuencia; Expresión facial; Piel; Rejuvenecimiento; Fisioterapia

RECEBIDO EM: 21/07/2021 APROVADO EM: 09/09/2021 


\section{artigo}

Santos, A. D., Santos, A. D., Vasconcelos, R. F. R., Bezerra, A. B

Fisioterapia dermatofuncional: radiofrequência e os efeitos no tratamento do rejuvenescimento da expressão da face

\section{AURICÉLIA DIAS SANTOS}

Fisioterapeuta, Especialista, Diretora do Instituto Holístico de Reabilitação - FisioNamaste. Universidade Estadual do CearaUECE.

ORCID: 0000-0002-0941-3878

\section{AURELIO DIAS SANTOS}

Fisioterapeuta, Mestre, Professor orientador deste artigo de pós-graduação, Coordenador do Instituto Holístico de Reabilitação - FisioNamaste. Centro Universitário Leão Sampaio - UNILEÃO, Universidade Cidade de São Paulo - UNICID. ORCID: 0000-0003-3693-9864

\section{ROSANGELA FROTA RIBEIRO DE VASCONCELOS}

Fisioterapeuta, Professora, Especialista e Coordenadora do curso de Fisioterapia da UNIJUAZEIRO. Centro Universitário de Juazeiro do Norte - UNIJUAZEIRO.

ORCID: 0000-0001-7511-0413

\section{ANA BEATRIZ BEZERRA}

Acadêmica de Fisioterapia. Centro Universitário Leão Sampaio. Acadêmica de Fisioterapia, ORCID: 0000-0002-0099-8427

\section{INTRODUÇÃO}

0 mercado mundial de Beleza e Estética está em constante crescimento e vem despertando interesse da área científica, devido às novas tecnologias para as diversas disfunçōes estéticas. A vaidade e a autoestima têm se destacado devido aos padrōes de beleza impostos pela sociedade e pela mídia. Portanto, aumentou as buscas por técnicas que atenuem e revitalizem as marcas de expressōes adquiridas com o tempo. Desta forma, caracterizando a necessidade e a importância do surgimento da área de especialização da fisioterapia dermatofuncional, objetivando a utilização de recurso e técnicas de tratamentos estéticos para promover a prevenção ou tratar sinais de envelhecimento como a flacidez e linhas de expressão.

$\mathrm{O}$ envelhecimento constitui-se em um processo complexo que afeta todos os órgãos e tecidos, sendo seus efeitos mais visivelmente observados na pele e nas estruturas subjacentes, originando alteraçōes estéticas e funcionais. Com o passar dos anos, a pele sofre alteraçōes degenerativas, perdendo a integridade mecânica da derme. Dessa forma em termos biológicos, o envelhecimento cutâneo caracteriza-se pelo achatamento da junção dermoepidérmica e pela atrofia geral da matriz extracelular com redução e desorganização do colágeno e elastina.
O envelhecimento

constitui-se em um

processo complexo

que afeta todos os

órgãos e tecidos,

sendo seus efeitos

mais visivelmente

observados na pele

e nas estruturas

subjacentes,

originando

alterações estéticas

e funcionais
O surgimento das linhas de expressão e flacidez são sinais que não necessariamente indicam a idade biológica do indivíduo, uma vez que o envelhecimento cutâneo depende de fatores intrínsecos e extrínsecos 3 . Fatores intrínsecos são aqueles relacionados com a idade e a genética, ocorrendo o desgaste natural do organismo que é o esperado e inevitável. Já os fatores extrínsecos estão relacionados com o frio, exposição solar, má alimentação, tabagismo e o estresse, favorecendo o aparecimento de sinais do envelhecimento mais evidentes.

$\mathrm{O}$ Avanço da tecnologia fundamentada pelas pesquisas cientificas evidenciam procedimentos de rejuvenescimento facial cada vez mais inovadoras que auxiliam na revitalização facial, ajudando no processo de envelhecimento da pele com vários recursos eletroterápicos e princípios ativos para o rejuvenescimento facial. Dentre os recursos, a Radiofrequência (RF) é um equipamento que se destaca ao produzir energia eletromagnética que é transformada em calor, aumentando a temperatura nos tecidos, produzindo nestes uma reação inflamatória que é capaz de promover o aumento da atividade dos fibroblastos, acarretando neocolagênese, neoelastogênese e remodelagem tecidual.

A radiofrequência é uma corrente de alta frequência que atinge a pele gerando calor controlado, na forma não ablativa. Promove calor por conversão entre $30 \mathrm{KHz}$ 
e $300 \mathrm{MHz}$, sendo a frequência mais utilizada entre 0,5 a $2,5 \mathrm{MHz}$ e a manutenção da temperatura em torno de $40^{\circ} \mathrm{C}$ durante o período de aplicação.

O calor fornecido nesse processo alcança as camadas mais internas do tecido cutâneo, acarretando contração das fibras e a formação de outras. Ocorre aumento da circulação sanguínea e redução de toxinas, o que causa uma melhora na oxigenação e ampliação do metabolismo, isso gera um tensionamento e tonificação instantâneos da pele.

Os efeitos fisiológicos da Radiofrequência são: vasodilatação, o que se dá por meio do aumento da circulação sanguínea, ocasionando também o movimento de nutrientes e oxigênio; atividade metabólica e enzimática, acarretada pelo aumento da temperatura; a viscosidade, pelo mesmo motivo anterior; alteração no tecido colagenoso; e estimulação nervosa, o que causa um efeito analgésico. Neste processo é importante o nível da hidratação da pele, pois quanto maior for, mais rápido ela retém o calor, haverá contração do colágeno, o qual terá sua síntese ampliada, um novo colágeno se formará, e esta dinâmica será progressiva às sessões de Radiofrequência.

Assim, o objetivo desta pesquisa é identificar, através de um levantamento bibliográfico, os efeitos da radiofrequência no rejuvenescimento facial.

\section{MÉTODOS}

Foi realizado o estudo de revisão integrativa bibliográfica, de caráter exploratório, observando-se o pressuposto da análise do levamento de achados científicos. A revisão integrativa consiste num método de pesquisa que tem como objetivo sintetizar os achados encontrados em bases de pesquisas, contextualizando um determinado tema ou questão. Tal processo, ocorre de forma ordenada e abrangente. Todavia, o revisor/pesquisador pode esquematizar uma revisão integrativa com diferentes abordagens, constituindo a viabilidade de definição, conceitos, revisão de teorias ou análise metodológica dos estudos elegíveis acerca de um tópico específico.
A caracterização da busca dos artigos nas bases de dados, foi realizada entre setembro e outubro de 2020 e constituída das seguintes fontes de pesquisas: Literatura Latino-Americana e do Caribe em Ciências da Saúde (LILACS), A Scientific Electronic Library Online (SciELO), Coordenação de Aperfeiçoamento de Pessoal de Nível Superior (CAPES) e PubMed. Para realização da busca dos artigos, utilizou-se o operador booleano "and" intercalado nos seguintes descritores: na língua portuguesa radiofrequência, expressão da face, pele Rejuvenescimento; Fisioterapia e para língua inglesa e Radio frequency; Face expression; Skin; Rejuvenation; Physiotherapy.

Os critérios de inclusão elegibilidade para seleção dos artigos foram: publicações em português e inglês; artigos na íntegra e gratuitos, artigos publicados e indexados nos referidos bancos de dados dos últimos 10 anos devido à escassez da especificidade do estudo. Foram excluídos artigos que não se relacionaram à temática da revisão integrativa e duplicados.

A classificação hierárquica das evi- dências, para a avaliação dos artigos dessa pesquisa, foi baseada na categorização da Agency for Healthcare Research and Quality (AHRQ). A qualidade das evidências é classificada em seis níveis: nível 1, (metanálise); nível 2 (estudo experimental); nível 3 (estudo quase-experimental); nível 4 (estudo não-experimental); nível 5 (relatório de estudos de casos); nível 6 (opinião de autoridades respeitáveis baseada na competência clínica).

A confecção da revisão integrativa seguiu as seguintes etapas orientadas, são elas: identificação do tema nas bases de pesquisa através da relação dos descritores, elegibilidade de critérios de inclusão/exclusão e artigos duplicados, conceituação das informações obtidas através da leitura de títulos e resumos, análise dos estudos escolhidos com leitura na integra, interpretação dos dados e apresentação da síntese do conhecimento apurado.

\section{RESULTADOS}

Realizou-se o cruzamento dos descrito-

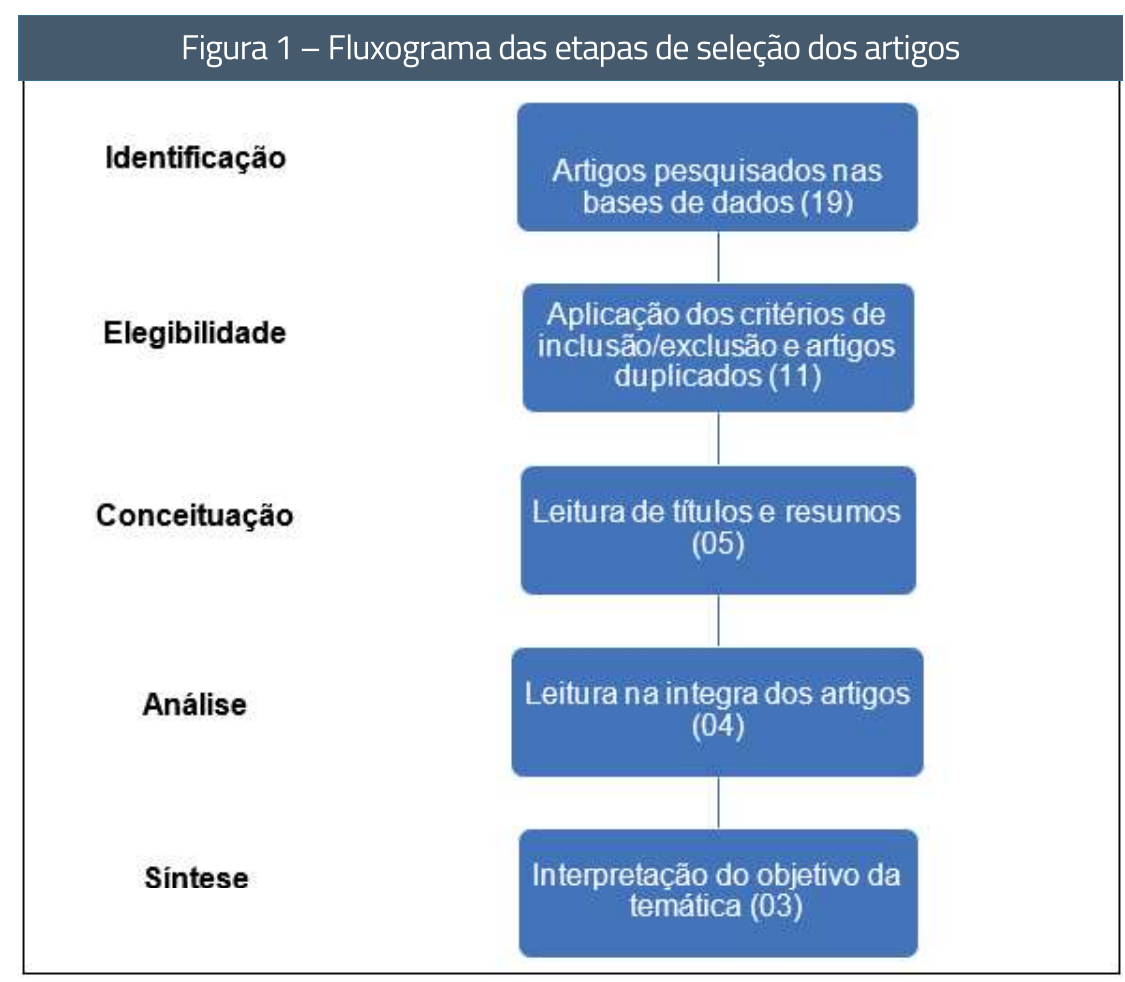

Elaborado pelo autor (2021). 


\section{artigo}

Santos, A. D., Santos, A. D., Vasconcelos, R. F. R., Bezerra, A. B.

Fisioterapia dermatofuncional: radiofrequência e os efeitos no tratamento do rejuvenescimento da expressão da face

res, ao que foram encontrados: 19 artigos na LILACS, em que apenas 2 artigos foram incluídos; 3 artigos na Scielo, porém nenhum foi incluído; 12 artigos no Portal da CAPES, com apenas 1 artigo inserido; e 09 artigos foram encontrados na plataforma PubMed, entretanto nenhum foi incluído. Dessa forma, a amostra foi constituída de 3 artigos, conforme observa-se na figura 1 .

A tabela 1 evidencia o nível de qualificação metodológica dos artigos segundo categorização da Agency for Healthcare Research and Quality (AHRQ). O qual percebe-se que a classificação dos artigos estabelece o mesmo nível 2 de qualidade metodológica satisfatória, conforme caracteriza a tabela 1 .

A tabela 2 caracteriza as principais informações de interpretação dos dados e apresentação da síntese do conhecimento apurado.

\section{DISCUSSÃO}

Um estudo 11, observou que, em relação aos resultados da satisfação dos pacientes quanto aos tratamentos testados, os aspectos investigados foram flacidez, rugas, textura e tonalidade da pele tratada. Para
Tabela 1 - Classificação hierárquica das evidências dos artigos

\begin{tabular}{|c|c|c|}
\hline ARTIGO & MÉTODO & $\begin{array}{c}\text { QUALIDADE } \\
\text { DAS EVIDÊNCIAS }\end{array}$ \\
\hline $\begin{array}{l}\text { DE LIMA GADELHA, Raissa de } \\
\text { Lima et al. Radiofrequência } \\
\text { pulsada para flacidez periorbitária: } \\
\text { estudo comparativo. Surgical \& } \\
\text { Cosmetic Dermatology, v. } 10, \text { n. 2, } \\
\text { p.140-146, } 2018 \text {. }\end{array}$ & $\begin{array}{l}\text { Estudo clínico } \\
\text { randomizado }\end{array}$ & Nível 2 \\
\hline $\begin{array}{l}\text { MARCHI, Juliana Pelissari et al. } \\
\text { Efetividade da radiofrequência } \\
\text { no tratamento facial de voluntá- } \\
\text { rias tabagistas e não tabagistas. } \\
\text { Arquivos de Ciências da Saúde da } \\
\text { UNIPAR, v. } 20 \text {, n. 2, } 2016 \text {. }\end{array}$ & Estudo clínico & Nível 2 \\
\hline $\begin{array}{l}\text { CASABONA, Gabriela et al. Radio- } \\
\text { frequência ablativa fracionada: um } \\
\text { estudopiloto com } 20 \text { casos para } \\
\text { rejuvenescimento da pálpebra } \\
\text { inferior. Surgical \& Cosmetic Der- } \\
\text { matology, v. 6, n. 1, pág. 50-55, } \\
2014 \text {. }\end{array}$ & Estudo clínico & Nivel 2 \\
\hline
\end{tabular}

\section{Tabela 2 - Delineamento, métodos e principais desfechos dos estudos selecionados}

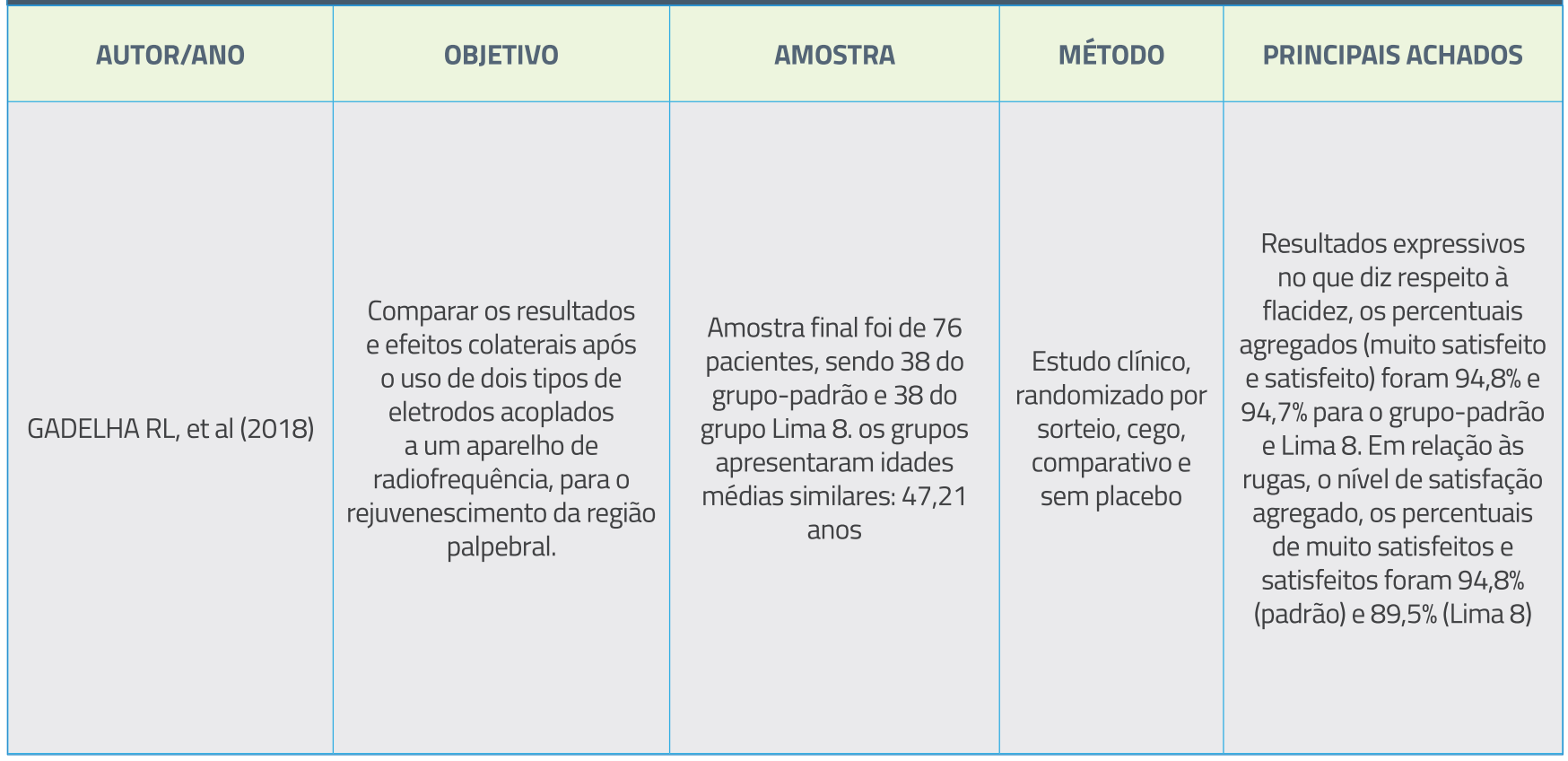




\begin{tabular}{|c|c|c|c|c|}
\hline MARCHI JP, et al (2016) & $\begin{array}{c}\text { Avaliar os efeitos do } \\
\text { tratamento facial com } \\
\text { radiofrequência em pele } \\
\text { de voluntárias tabagistas e } \\
\text { não-tabagistas. }\end{array}$ & $\begin{array}{l}8 \text { voluntárias do sexo } \\
\text { feminino, sendo } 04 \\
\text { tabagistas e } 04 \text { não- } \\
\text { tabagistas, com idade } \\
\text { entre } 47 \text { e } 53 \text { anos }\end{array}$ & $\begin{array}{l}\text { Estudo clinico } \\
\text { comparativo }\end{array}$ & $\begin{array}{l}\text { os participantes } \\
\text { do estudo apresentaram } \\
\text { redução na extensão } \\
\text { das rugas medidas pelo } \\
\text { paquímetro na face } \\
\text { direita. A ANOVA de duas } \\
\text { vias revelou alterações } \\
\text { significativas após } 10 \\
\text { sessões de } \\
\text { tratamento }(F 3,22=3,39 ; \\
\qquad<0,05)\end{array}$ \\
\hline CASABONA G, et al (2014) & $\begin{array}{l}\text { Descrever princípios } \\
\text { de funcionamento, } \\
\text { metodologia e resultados } \\
\text { do rejuvenescimento } \\
\text { em pálpebras } \\
\text { inferiores tratadas com } \\
\text { radiofrequência ablativa } \\
\text { fracionada e consequente } \\
\text { dano termal observado na } \\
\text { histopatologia }\end{array}$ & $\begin{array}{c}\text { Foram selecionadas } \\
\text { aleatoriamente } 20 \\
\text { pacientes. Idade entre } 40 \text { e } \\
65 \text { anos, do sexo feminino }\end{array}$ & $\begin{array}{l}\text { Estudo clínico } \\
\text { prospectivo }\end{array}$ & $\begin{array}{c}\text { Das } 20 \text { pacientes, } 18 \\
\text { ficaram muito satisfeitas } \\
(90 \%) \text { e apenas duas ( } 10 \%) \\
\text { apenas satisfeitas com os } \\
\text { resultados de melhora da } \\
\text { textura e flacidez da pele. }\end{array}$ \\
\hline
\end{tabular}

Elaborado pelo autor (2021).

flacidez, $57,9 \%$ dos pacientes submetidos ao tratamento com ponteira no grupo B relataram estar muito satisfeitos, enquanto esse percentual no grupo-padrão foi de $55,3 \%$ dos pacientes ficaram satisfeitos com os resultados proporcionados pelo tratamento no grupo-padrão e $36,8 \%$ no grupo B. De modo geral, ambos os tratamentos apresentaram resultados expressivos no que diz respeito à flacidez, visto que os percentuais agregados (muito satisfeito e satisfeito) foram $94,8 \%$ e $94,7 \%$ para o grupo $\mathrm{A}$ e grupo $\mathrm{B}$, respectivamente. Com relação às rugas, mais da metade dos pacientes estão satisfeitos com ambos os tratamentos analisados, cujos percentuais foram $73,7 \%$ para o grupo-padrão e $50 \%$ para o grupo B. Avaliando o nível de satisfação agregado, os percentuais de muito satisfeitos e satisfeitos foram 94,8\% (grupo A) e 89,5\% (grupo B).

Portanto, observa-se que em relação ao grupo padrão que utilizou o aparelho de radiofrequência convencional, que faz jus a nossa pesquisa, os resultados delinearam-se de maneira bastante evidente, principalmente no que diz respeito a melhora do tônus da pele e da diminuição das rugas.

Percebe-se que o grupo tabagista e não-

\section{Os autores}

ressaltam que a

radiofrequência

constitui mais uma

possibilidade para

o tratamento do

envelhecimento

cutâneo -tabagista apresenta características semelhantes em relação ao tamanho da ruga presente no canto externo do olho, e, portanto, não foram encontradas diferenças significativas entre os grupos (F 1,22 = 2,36; p = 0,13 ), o que demonstra a homogeneidade entre os grupos em estudo. Todos os participantes do estudo apresentaram redução na extensão das rugas medidas pelo paquímetro na face direita. A ANOVA de duas vias revelou alterações significativas após 10 sessões de tratamento (F 3,22 = 3,39; p < 0,05).

A análise com o post-hoc de Duncan demonstrou que houve diferença significativa quando comparamos a $1^{\text {a }}$ e a $10^{\text {a }}$ sessão das voluntárias não-tabagistas $(\mathrm{p}<0,05)$. A mesma diferença estatística foi encontrada quando comparamos a $1^{\text {a }}$ sessão do grupo tabagista com a $10^{\mathrm{a}}$ sessão do grupo não-tabagista. Os autores desse estudo comentam que os efeitos da radiofrequência são significativos na redução da extensão e profundidade das rugas e na melhora da aparência da flacidez pele, além de melhora perceptível da aparência facial, ou seja, é um tratamento completo para revitalização cutânea. 


\section{artigo}

Santos, A. D., Santos, A. D., Vasconcelos, R. F. R., Bezerra, A. B.

Fisioterapia dermatofuncional: radiofrequência e os efeitos no tratamento do rejuvenescimento da expressão da face

Em outro achado12, das 20 pacientes, 18 ficaram muito satisfeitas (90\%) e apenas duas (10\%) apenas satisfeitas com os resultados. Duas (10\%) apresentaram hiperpigmentação pós-inflamatória da região tratada, que se resolveu após o uso da combinação hidroquinona/tretinoína tópica durante 15 dias. Os autores ressaltam que a radiofrequência constitui mais uma possibilidade para o tratamento do envelhecimento cutâneo. Devido caracterizar-se por seu um procedimento que emite ondas que alcançam as camadas mais profundas da pele, gerando sobre elas energia e forte calor, porém mantendo a superfície resfriada e protegida. Faz-se necessário enfatizar que os pesquisadores relatam que os achados deste estudo, demonstrou que essa técnica pode ser evidenciada como um tratamento eficaz para o rejuvenescimento da região periorbitária. Desta forma, observa-se que atualmente, existem vários estudos em andamento tentando demonstrar esses efeitos não só nas pálpebras, mas em toda a face, em cicatrizes de acne, cicatrizes inestéticas e estrias.

\section{CONCLUSÃO}

Após a revisão dos achados encontrados e analisados os consensos das literaturas, no que se diz respeito aos efeitos da radiofrequência para o tratamento estético da pele, especificamente na região do rosto. Podemos perceber que se trata de um método terapêutico bastante utilizado na fisioterapia, na especialidade de dermatofuncional. $\mathrm{O}$ qual demonstrou que os objetivos propostos foram alcançados, os quais foram caracterizados pelos efeitos eficazes e seguros na redução de rugas e flacidez cutânea da região da face.

Observa-se que o objetivo alcançado nesta revisão, pode ser de relevância e aplicabilidade para comunidade, uma vez que consiste numa abordagem terapêutica segura, de fácil acesso econômico e que não promove efeitos colaterais na pele, pois não tem aplicabilidade invasiva.

Todavia, há poucos estudos disponíveis na integra, principalmente de natureza gratuita sobre esse assunto. Portanto, para tal dificuldade de acesso a estudos de ensaios clínicos com boa qualidade metodológica e gratuitos. Recomenda-se a importância da realização de novos estudos clínicos randomizados e de acesso livre para comunidade acadêmica e sociedade. Fundamentado, desta forma, o acesso fácil das atualizaçōes cientificas sobre a efetividade cientifica da radiofrequência no tratamento de rugas e flacidez da pele.

\section{REFERÊNCIAS}

1. Macedo MCA, Tenório CA. Tratamento de rugas: uma revisão bibliográfica sobre carboxiterapia, radiofrequência e microcorrente. Vis Univ, Cassilândia, mar 2015; 2, 1-20.

2. Tassinary J, Sinigaglia M, Sinigaglia G. Raciocínio Clínico aplicado à estética facial. Edit Estét Exp. 2019. $1^{\text {a }}$ Ed. Lajeado/RS.

3. Silva MVR, Hansen, D, Sturzenegger, TM. Radiofrequência no Rejuvenescimento Facial. Ciên, Reflex e Incert. Cruz Alta, nov 2012;:2: 1-6.

4. Cruz EF. et al. Radiofrequência no tratamento das rugas faciais. Rev da Univ Ibirapuera, São Paulo, jun. 2014; 7: 38-50.

5. Fonseca E, Alves J, Hasse R. O uso da Radiofrequência no tratamento de rejuvenescimento facial. Rev Estét em Mov. Universidade FUMEC. 2018; 1(1):1-17.

6. Lofeu GM. Atuação da radiofrequência na gordura localizada no abdômen: revisão de literatura. Rev da Univ Vale do Rio Verde، Três Corações, jun 2015; 13(1): 571-581.

7. Silva, SCP. et al. Radiofrequência associada à drenagem linfática manual no rejuvenescimento facial. Saúde Coletiva (Barueri). 2020; 10(55),: 2765-2780

8. Duarte, AB.; Mejia, DPMA. utilização da Radiofrequência como técnica de tratamento da flacidez corporal. Portal Biocursos [internet]. 2012 [acesso em: 2020 set 19]; Disponivel em: https://
portalbiocursos.com.br/ohs/data/docs/19/35_-_A_utilizaYYo da_RadiofrequYncia_como_tYcnica_de_tratamento_da_flacidez_corporal.pdf

9. Ercole FF, Melo LS, Alcoforado CLGC. Revisão integrativa versus revisão sistemática. Rev Mineira de Enf. 2014; 18(1); 9-12.

10. Galvão CM, Sawada NO, Mendes IAC. A busca das melhores evidências. Rev da Esc de Enf da USP. 2003; 37(4): 43-50.

11. Gadelha RL et al. Radiofrequência pulsada para flacidez periorbitária: estudo comparativo. Surg \& Cosm Derm,. 2018;10(2): 140-146.

12. Marchi JP et al. Efetividade da radiofrequência no tratamento facial de voluntárias tabagistas e não tabagistas. Arq. de Ciênc da Saúde da UNIPAR. 2016; 20(2).

13. Casabona $\mathrm{G}$ et al. Radiofrequência ablativa fracionada: um estudopiloto com 20 casos para rejuvenescimento da pálpebra inferior. Surg \& Cosm Derm. 2014; 6(1): 50-55. 